\title{
S)

\section{A Case Study Approach to Train Early-Stage Investigators in Transdisciplinary Research}

\author{
Emily C. Benesh, PhD ${ }^{* 1, a-b}$; Laura E. Lamb, PhD ${ }^{* 2, c-d}$; Shahnjayla K. Connors, PhD, MPH, \\ $\mathrm{CPH}^{3, e}$; Grant W. Farmer, PhD, MPH, MA ${ }^{4, a}$; Katherine C. Fuh, MD, PhD ${ }^{5, f}$; Jean Hunleth, \\ PhD, MPH ${ }^{4, e}$; Katherine L. Montgomery, PhD, MSSW ${ }^{6, g}$; Alex T. Ramsey, PhD, MA ${ }^{6, g}$; \\ Kelle H. Moley, MD ${ }^{1, a}$; Graham A. Colditz, MD, DrPH ${ }^{4,7, a}$; Sarah J. Gehlert, PhD $\mathbf{D}^{4,5,7, a, e, h, i * *}$ \\ ${ }^{1}$ Divison of Basic Sciences Research, Department of Obstetrics and Gynecology Washington University School of Medicine in \\ St. Louis, Saint Louis, MO; ${ }^{2}$ Beaumont Health Systems Research Institute, Royal Oak, Ml; ${ }^{3}$ The Genome Institute at \\ Washington University School of Medicine in St. Louis, Saint Louis, MO; ${ }^{4}$ Division of Public Health Sciences, Department of \\ Surgery, Washington University School of Medicine in St. Louis, Saint Louis, MO; ${ }^{5}$ Divison of Gynecologic Oncology, \\ Department of Obstetrics and Gynecology, Washington University School of Medicine in Saint Louis, Saint Louis, MO; \\ ${ }^{6}$ George Warren Brown School of Social Work, Washington University in St. Louis, Saint Louis, MO; ${ }^{7}$ Siteman Cancer Center, \\ Washington University School of Medicine in St. Louis, Email: sgehlert@wustl.edu.
}

Personal Acknowledgement:We wish to dedicate this manuscript to our departed colleague Dr. Grant Farmer. His work and inspiration were essential to the genesis of these ideas and the construction of this manuscript.

\section{doi: $10.22545 / 2015 / 00071$}

T ransdisciplinary (TD) research is a collaboration in which investigators from diverse backgrounds co-generate ideas. Few successful examples of TD research outcomes have been reported, possibly due to a training barrier. Here, $T D$ trainees present a case study methodology that augmented classic training exercises by removing hierarchical barriers and allowing the practice of TD methodologies. A 30-minute development period was critical for the team to enter the conceptualization phase of TD research, making 90-minute sessions preferable for these exercises. Six sessions over an academic year were necessary for optimum idea formation. Generating buy-in was a challenge, as pressuring potential team members to participate would alter viewpoint equitability.
Internal and external enthusiasm grew over the time period. Participation led to sustained collaborations and provided a marketable skillset. This method was low-cost and, likely, generalizable to other institutions. Thus, case study approaches may be effective tools to train researchers in TD interactions.

Keywords: transdisciplinary research, postdoctoral fellows, training, case studies.

\section{Introduction}

Transdisciplinary (TD) research is a mode of collaboration in which investigators operate outside their disciplines to generate shared research aims [1]. The overarching goal of TD research is to re- 
Emily C. Benesh, Laura E. Lamb, Shahnjayla K. Connors, Grant W. Farmer, Katherine C. Fuh, Jean Hunleth, Katherine L. Montgomery, Alex T. Ramsey, Kelle H. Moley, Graham A. Colditz, Sarah J. Gehlert

A Case Study Approach to Train Early-Stage Investigators in Transdisciplinary Research

duce latency periods between discovery of potential therapeutic tools and their implementation in the larger population [2]. The National Institutes of Health have identified the need for TD research and projects such as the Washington University in St. Louis Transdisciplinary Research on Energetics and Cancer program (WUSTL-TREC) and the Program for the Elimination of Cancer Disparities (PECaD) have been funded to build infrastructures to support these efforts [3-6]. TD research is valuable because it allows a group to collectively: 1) identify complex public health concerns that would benefit from the expertise of a cross-disciplinary team, 2) systematically elucidate causative agents, 3) design multi-level effective interventions/treatments, 4) determine how best to implement such efforts in affected communities, and 5) implement them in a timely manner, while working within the framework of government, business, and advocacy groups [6]. The TD process requires that team members understand and exercise skill sets from other disciplines in which they lack formal training, thus trust must be built among team members across disciplines. Because collaborations across divergent disciplines (e.g. anthropology and molecular biology) are unusual, training scientists in the skills required for TD science is critical.

As observed in a classic reference on organizational change, The Heart of Change: "People will think of themselves or of their subgroups first and be protective and suspicious (of others) [7]." For this and other reasons, such as hiring and tenure and promotion, TD research is neither intuitive nor easy. Barriers to TD have been expertly reviewed and include: high labor intensity, lack of interdisciplinary understanding and subsequent conflicts, difficulty for team members to learn a common language, need for shared infrastructure, and the expense of associated costs [8]. Traditional training does not equip scientists with skill sets to mitigate these challenges. These have made examples of effective TD research relatively rare [2], and have highlighted the need to augment traditional training methods $[8,9]$.

Traditional TD training is based on a multi-mentor apprenticeship model that provides trainees with the following resources: exposure to the process of TD thinking, an expanded scientific lexicon, preparation for career advancement challenges, and protective measures to prevent regression to single disciplinary methodologies [9]. WUSTL-TREC and others have established training programs that achieve these goals. These programs use classical training methodologies such as formal seminars, journal clubs, didactic classes, and individual-project presentations $[8,9]$. The training garnered by WUSTL-TREC introduced our team to a knowledge-base and comfort that supported team members' interactions with others' disciplines, began to establish a shared lexicon, provided career development preparation, and fostered small collaborations. However, we observed a disconnect between gaining knowledge of the TD process and developing the ability to effectively participate in or lead TD research independently of WUSTL-TREC.

We argue here that a critical gap in TD training is often missing: the practice of TD research methodologies by assessing public health issues and brainstorming research approaches in a bias-free environment with a team of engaged peers from diverse disciplinary backgrounds. We suggest that a case study approach using team-based language and mentality is an effective method to practice and train in this skill set. In our initial training, our team of seven postdoctoral fellows and one early career investigator experienced a formality and overlying power structure inherent in a traditional didactic training style that granted authority to the discipline of that session's leader. This led to disciplinary-specific discussions and inhibited the creative process required for genuine TD idea generation. For example, if the leader was an epidemiologist, then the training session was taught in and flowed from an epidemiological mindset. Additionally, we encountered some aforementioned TD barriers, such as problems with communication across disciplines. We found that with traditional training methods, the leading discipline was often the final word in conflict resolution, potentially biasing a discussion.

In our case study experience, we initially observed significant challenges to buy-in and idea generation during a case study. Thirty minutes of an introduction period, herein described as "The Thirty Minute Rule", occurred before true collaborative idea generation. This required that case study sessions last at least 90 minutes. Team members: 1) were fully involved, from case study topic selection to postdiscussion reflection, 2) solved problems together and generated TD ideas for future work, 3) developed a shared lexicon, 4) mediated arguments in an open forum, and 5) applied TD-related concepts outside of the allotted discussion times. The re- 
Emily C. Benesh, Laura E. Lamb, Shahnjayla K. Connors, Grant W. Farmer, Katherine C. Fuh, Jean Hunleth, Katherine L. Montgomery, Alex T. Ramsey, Kelle H. Moley, Graham A. Colditz, Sarah J. Gehlert

A Case Study Approach to Train Early-Stage Investigators in Transdisciplinary Research

sults were open communication around a given topic, long-term interdisciplinary collaborations, and development of a valuable skill set. Furthermore, our method was low-cost. Thus, we offer that case study approaches are effective tools to train researchers in TD interactions and may be applicable to other institutions.

\section{Developing the TD Team and Lessons Learned}

Here we describe a framework for a trainee-run case study TD training approach, including the steps underwent to launch it, barriers we experienced, and lessons learned.

\section{1) Assessing institutional preparedness for a} TD effort - Stokols and colleagues succinctly and thoroughly defined characteristics of institutional collaborative-readiness including: institutional support, a wide breadth of disciplines housing trainees, a high degree of prior team cooperation, the availability of convenient meeting places and/or close spatial proximity between collaborators, and the availability of electronic communication tools [8]. Because of WUSTL-TREC, our institution was highly collaborative-ready.

2) Recruiting advisors - Organizing members (ECB and LEL) first approached supportive mentors (KHM, GC, SG), who provided ideas and infrastructure. We also sought topical expertise. Dr. Julie Turner (Van Andel Institute) provided insight on leveraging case studies for TD training, structuring the team, and establishing language to foster trust and openness. Dr. Doug Larsen (Washington University) made suggestions on general team structure and training evaluation.

3) Generating buy-in - Team members were faced with expending time to an endeavor that might not yield immediate career-building results. Departmental, mentor-based, or other compulsive pressures threatened to negate efforts to build a foundation in which the power structure was equalized. Therefore, buy-in had to be generated at the outset.

Generating initial buy-in was difficult. Fifteen potential postdoctoral fellows and early stage investigators were asked to participate and only five initially accepted. Although we do not know why some declined, opinions expressed included concern about the need for great time and effort, that it was messy with an undefined endpoint, and could potentially expose individual gaps in knowledge. Initial reticence to participate was also attributed to a lack of clarity about outcomes of the process, difficult travel considerations, and being more distantly related to the existing TREC infrastructure. Trainees who chose not to participate likely also had a range of concern related to career stage, home life, and timing. Our case study team grew from a strong existing WUSTL-TREC infrastructure that supported the time spent on this endeavor, yet we still experienced this barrier. Thus, we suggest that generating buy-in is a challenge that might be experienced by many initiate teams. Interestingly, the testament of the early adopting members fostered increased recruitment; members assured others that use of the TD methodology was being achieved and our final team included eight members. Once a team member participated in one TD discussion session any hesitancies lessened, and no attrition was experienced. Validation of the importance of this effort was given by WUSTL-TREC leadership and outside support (e.g. the external advisory board). Thus, non-compulsory buy-in can occur both through initial and secondary recruitment. Conversely, buy-in could not be coerced from uninterested participants. An academic year was required to achieve a wellrepresented, cohesive team.

4) Team Composition and Career Stage - Our final team was composed of seven postdoctoral fellows and one investigator in her first year as assistant professor. Effort was made to minimize overrepresentation of one discipline over others although team members were self-selected. Most initial recruits were trained in the basic sciences (developmental biology, cancer biology, and molecular biology with public health training) or clinical sciences (gynecological oncology). Also, one initial member was a social epidemiologist. As successful discussions occurred, three additional social scientists asked to join (specializing in anthropology, behavioral health, and implementation science). None of the members withdrew from the team during the training.

While our methodology may provide value-added TD training for researchers at all career stages, our experience suggested that three criteria are needed: 
Emily C. Benesh, Laura E. Lamb, Shahnjayla K. Connors, Grant W. Farmer, Katherine C. Fuh, Jean Hunleth, Katherine L. Montgomery, Alex T. Ramsey, Kelle H. Moley, Graham A. Colditz, Sarah J. Gehlert

A Case Study Approach to Train Early-Stage Investigators in Transdisciplinary Research

1) expertise and confidence in one's own field;

2) time for additional training activities; and

3) an openness to the value and limits of any singular discipline that may be intrinsic or taught from previous training experiences (as was done for our members by the existing WUSTL-TREC and PECaD infrastructures).

Postdoctoral fellows may be particularly amenable to the transformations intended to occur during TD training because of their transitional status. They are at a career stage in which they are determining specific questions and career paths for future work. We found that this transitional state opened our behaviors to new disciplinary perspectives rather than operating from preset rules of thinking; a characteristic necessary for effective TD research. Also, some team members were considering TD research as a potential de facto career option. Postdoctoral fellows in the middle of their training had the most flexibility in time and effort and, thus, willingness to dedicate time to the TD training exercises. While this timing-related "sweet spot" may differ from one TD group to the next, it is worth considering in the planning stages in order to maximize the success of TD training groups.

5) Civility and conflict management - A commonly cited challenge to TD research is the need for mediation when disciplinary perspectives come into conflict $[8,10]$. We addressed this by generating civility guidelines at the outset, which included: 1) respect for all disciplines, 2) sincerity in all comments, 3) allowing respectful requests to table vignettes, and 4) time management. Because the rules were self-developed and enforced, no power structure alterations seemed to be introduced. Importantly, we encountered little need to mediate arguments as trust was built. Regular solicited feedback provided the members opportunity to voice concerns.

Authorship of publications was discussed in advance. It was agreed that the co-first authors had contributed most to the generation of ideas and would retain the major responsibility for synthesizing the paper; a coin-toss determined their order. Middle authors were ranked alphabetically and the last three authors were the established mentors who supported the effort. This largely reflects the convention of basic science, where the senior author comes last, as opposed to social sciences, where authors are ranked in order of effort.

These characteristics might be best embodied in an early career pool of members (e.g. postdoctoral fellows and early career investigators). When adequately supported by their mentoring teams, postdoctoral fellows may have more freedom to undertake TD training than more senior researchers, who face additional pressures.

6) Case study approach - We chose a case study approach to address the critical gap in TD training (i.e. trainees must practice doing TD). Case study training programs are team-building exercises that allow members to negotiate different problemsolving skills in new ways, while retaining focus within a specific context [11]. As such, case studies allowed our members to apply their expertise to novel concepts and work across disciplines to design approaches and generate solutions within a given study. The initiators of the team brainstormed examples of potential topics for the first two sessions; the team brainstormed the subsequent topics. Our team worked through six case studies that were intentionally broad-based and arose from a common research interest in cancer. Topics ranged from known to unknown etiology (Table 1). It was critical to work through several case studies because particular topics organically focused discussions toward certain disciplinary mindsets and methodologies.

Discussions were held monthly for 90 minutes over a period of eight months. Related articles were offered prior to meetings. During the discussion, team members were asked to form hypotheses and design methods to address specific questions, but the conversation was intentionally free flowing with minimal mediation (general discussion flow outlined in Fig. 1a). The last five minutes were spent summarizing the discussion.

Throughout the process, the team's communication, trust, and ability to generate ideas increased markedly. For example, the second case study involved prostate cancer disparities in African American men. Few TD ideas emerged from this early discussion. Rather, the team learned to discuss sensitive topics, perform ad hoc research during the meeting, and overcome unfamiliarity with working as a team. We also determined points at which external experts were required (outlined in Fig. 1a). By the last meeting, many of these initial teamwork 
Emily C. Benesh, Laura E. Lamb, Shahnjayla K. Connors, Grant W. Farmer, Katherine C. Fuh, Jean Hunleth, Katherine L. Montgomery, Alex T. Ramsey, Kelle H. Moley, Graham A. Colditz, Sarah J. Gehlert

Table 1: Case Study Topics Ranging from Known to Unknown Etiologies

\begin{tabular}{|l|l|}
\hline Case Study Topic Types & Specific Discussion Topics \\
\hline $\begin{array}{l}\text { Examples of collaborative efforts to determine } \\
\text { etiology and interventions }\end{array}$ & Lung cancer and smoking \\
\hline $\begin{array}{l}\text { Existing databases on disease clusters and risk } \\
\text { factors }\end{array}$ & $\begin{array}{l}\text { African American disparities in prostate cancer in } \\
\text { Michigan }\end{array}$ \\
\hline Mechanisms of widespread diseases & $\begin{array}{l}\text { Transmission of Human Papilloma Virus (HPV) } \\
\text { and cervical cancer }\end{array}$ \\
\hline $\begin{array}{l}\text { Potential causal agents and disease risk in } \\
\text { populations }\end{array}$ & $\begin{array}{l}\text { (1) Social barriers to HPV vaccination rates } \\
\text { (2) Substance use and adolescent delinquency }\end{array}$ \\
\hline $\begin{array}{l}\text { Local public health concerns of interest shared by } \\
\text { group members }\end{array}$ & $\begin{array}{l}\text { Local waste fire near nuclear waste dumping } \\
\text { ground }\end{array}$ \\
\hline
\end{tabular}

a. Case Study Approach
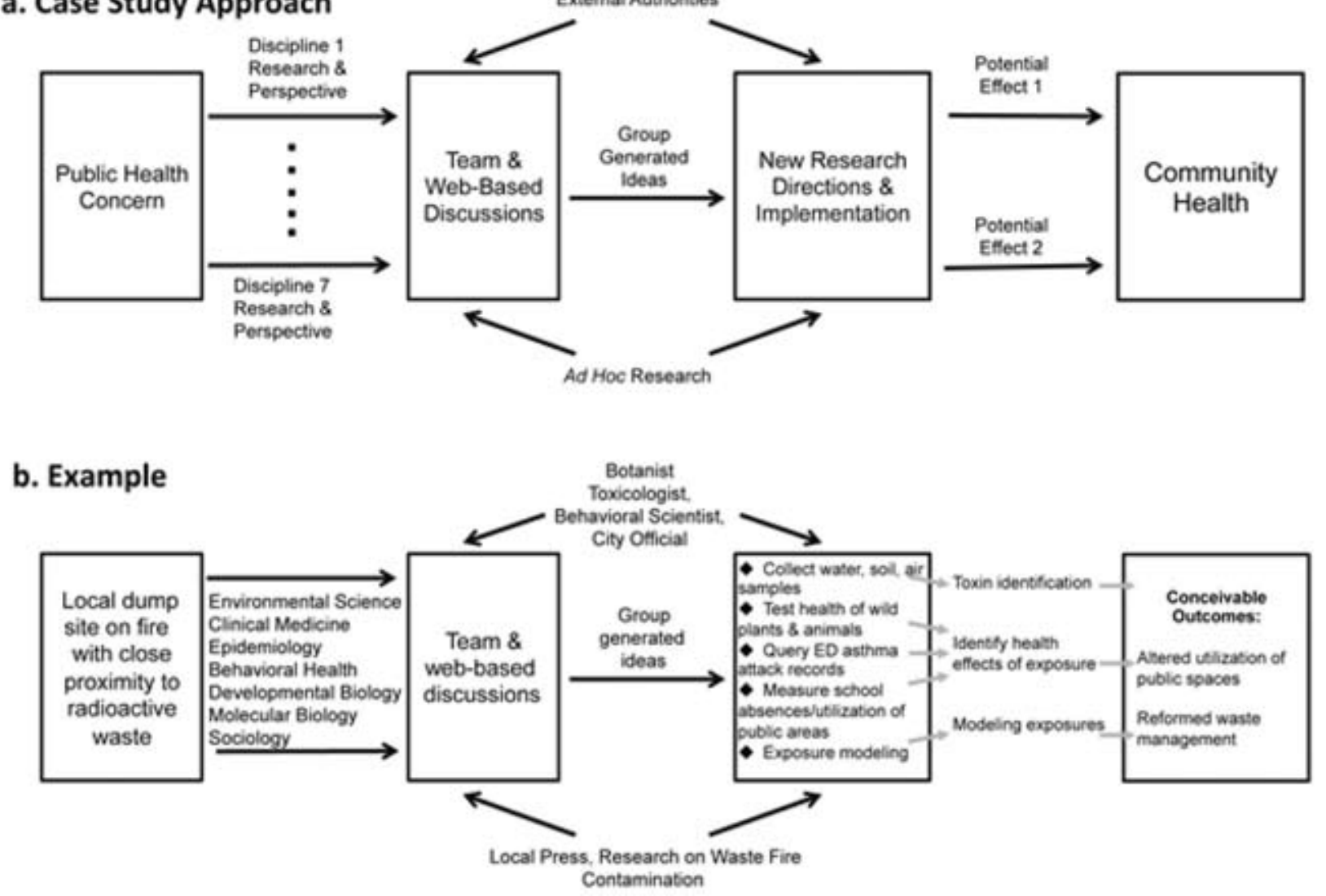

Figure 1: a) A diagram of the intended workflow of a transdisciplinary case study meeting. Team members choose a public health topic, perform discipline-specific research and bring their unique perspective to both in-person and follow-up web-based meetings. During these meetings and subsequent discussions, opinions are gathered from outside experts and additional research on topics is done as needed. Future discussions would determine a workflow for chosen research directions and implementation with the goal of affecting the community in need. This is not based on measurement. b) An example of outcomes from one case study experience.

based barriers had been resolved. Our final case study was on a local waste site on fire. During this discussion, the team developed several approaches to investigating whether the fire had adverse health effects in the surrounding community. Fig. 1b gives an outline of ideas and directions generated from this 
Emily C. Benesh, Laura E. Lamb, Shahnjayla K. Connors, Grant W. Farmer, Katherine C. Fuh, Jean Hunleth, Katherine L. Montgomery, Alex T. Ramsey, Kelle H. Moley, Graham A. Colditz, Sarah J. Gehlert

A Case Study Approach to Train Early-Stage Investigators in Transdisciplinary Research

last discussion. Successful completion of such studies indicating effects on health risks has the potential to inform city planning, waste site management, and public policy.

7) The 30-Minute Rule - Primary challenges to effective TD research include how to navigate discipline-specific communication styles, understand discipline-specific terms and create a shared lexicon, develop shared research objectives, and jointly conceptualize scientific problems [12]. We sought to navigate these barriers with the case study approach. Previous studies have proposed that TD research transpires in four phases: development, conceptualization, implementation, and translation [12]. This model applied to our case study discussion experiences. The first approximately 30 minutes of each case study was devoted to defining the topic through the lens of each representative field, referred to as the development phase [12]. We found that regardless of topic, a shift in mutual understanding occurred after 30 minutes. At this point, the team entered the conceptualization phase and began to generate novel ideas. We referred to this as the "30-Minute Rule." As the sessions progressed throughout the year and a shared lexicon was developed, this lag period shortened slightly and the amount of ideas generated in the developmental phase increased. Therefore, we found that team members must persevere through initial communication barriers during the development phase and be willing to meet for 90 minutes. We also provided opportunities for team members to continue discussions outside of meetings (e.g. an online forum). Because many classical training methodologies do not require lengthy latency periods before ideas are generated, if unanticipated, this lag period could be demoralizing for new initiate TD case study teams.

8) Benefits of Participation - Long-term collaborations: An unanticipated finding of this exercise was the development of new cross-disciplinary collaborations between team members. For example, a basic scientist and an epidemiologist initially investigated entirely disparate topics. Yet, a new collaboration arose in which epidemiological expertise was utilized to assess human survey data relating to findings identified in animal models. Survey data would then reciprocally inform the development of future animal studies. Collaborations such as these are unique be- cause the two disciplines came together to generate the research idea and methodology of study, as opposed to one discipline using another to achieve the individual aim of the initiating discipline. Because the research questions were generated together, the scope of the project was broad. Yet the ability to anticipate disciplinary pitfalls added to feasibility of the study. As team members formed long-term relationships during the case study journey, they felt comfortable asking for each others' expertise and thus anticipated many future collaborations.

Marketable Skills: Postdoctoral fellows on the job market found that potential employers favorably viewed their team-based experiences. Indeed, an evolution of attitudes within the team seemed to progress towards open-mindedness over time. Team members exhibited a high willingness to engage with one another so that collaborations matured over the course of the year. By the end of the term, members existed as a team rather than individual experts. At the conclusion of each case study interaction, team members anecdotally reported high feelings of energy and optimism around the topic and ideas generated. The evidence of collaborative skills gained during this process was easily leveraged into leadership and cooperation talking points during interviews.

Direct measures: The effectiveness of TD interactions is often measured by co-authorships on manuscripts and grant proposals [13]. As we are only a few months removed from this experience, these metrics are premature. However, confidence in team members by WUSTL-TREC mentors has increased as reflected by additional TD opportunities offered to our team members including: national meeting oral presentations, manuscript authorships, and investigator status (rather than trainee) on projects and grants.

9) Plans for Sustainability - As our team members move on to other positions or encounter careerstage pressures, sustainability of this specific collaboration will be a challenge. However, there is optimism that case study-based TD training initiatives will be sustainable within the WUSTL organization as TD infrastructures could provide a pool of candidates for future teams. We anticipate that the skills developed in the case study process will transfer to future TD endeavors. The case study team has achieved acknowledgement from institutional authorities, which gives it credibility. To ensure sus- 
Emily C. Benesh, Laura E. Lamb, Shahnjayla K. Connors, Grant W. Farmer, Katherine C. Fuh, Jean Hunleth, Katherine L. Montgomery, Alex T. Ramsey, Kelle H. Moley, Graham A. Colditz, Sarah J. Gehlert

A Case Study Approach to Train Early-Stage Investigators in Transdisciplinary Research

tainability, proof-of-principle recommendations for a problem identified by the team need to be published. Determining whether a team member number ceiling exists for this training methodology is important. Additionally, the case study process will be refined by presenting the project at national meetings.

\section{Conclusions}

In conclusion, TD research is an exciting opportunity for teams of researchers to leverage their training differences to shorten the latency between intervention discovery and implementation in the community TD research has historical barriers that require training to overcome. We suggest that case study based practice approaches can limit inherent power structures that disrupt equitable idea generation that is present in other training forums. The approach described here is particularly well suited to postdoctoral fellows and early stage investigators. We found that a 30-minute development phase was needed before conceptualization and idea generation became prolific. Our case study approach fostered open, authentic communication in a safe environment where team members engaged in problem solving. This technique was low cost, making it a potential training opportunity for all institutions interested in TD research.

\section{Acknowledgments}

We thank Dr. Julie Turner (Van Andel Institute) for initial discussions on how to leverage case studies for TD training and how to structure the group and language used to foster trust and openness.

\section{Grant Funding}

Drs. Benesh and Farmer were supported by the Washington University Transdisciplinary Research in Energetics and Cancer Program Grant (NCI; U54 CA 155496, PI: Colditz). Dr. Benesh was also supported by the Washington University Department of Pathology Training Grant (5T32 DK 7296-33, PI Colonna).

Drs. Connors and Hunleth were supported by Program for the Elimination for Cancer Disparities (PECaD) (National Institutes of Health-National
Cancer Institute Community Network Program, U54 CA153460; Colditz, PI).

Dr. Lamb was supported by the Department of Defense Prostate Cancer Postdoctoral Training Award (W81XWH-12-1-0119) and by the Washington University Center for Women's Infectious Disease Research.

Dr. Fuh is an NIH/NICHD Scholar, Reproductive Scientist Development Program (2K12HD000849-26, PI: Moley).

Drs. Ramsey and Montgomery are supported by the Mental Health Services Postdoctoral Training Program (NIMH T32 MH 019960, PI: Raghavan).

Dr. Moley is the James P. Crane Professor and Vice-Chair and Director of Basic Science Research in the Department Obstetrics and Gynecology at the Washington University School of Medicine and was supported by U54 CA 155496.

Dr. Colditz is the Niess-Gain Professor of Surgery, Professor of Medicine, the Chief, Division of Public Health Sciences, Department of Surgery, Washington University School of Medicine and the Associate Director Prevention and Control, Alvin J. Siteman Cancer Center. For this project he was supported by U54 CA153460 and U54 CA 155496.

Dr. Gehlert is the E. Desmond Lee Professor in the George Warren Brown School of Social Work, Professor, Department of Surgery at the Washington University School of Medicine, and Co-director of the Prevention \& Control Program in the Siteman Cancer Center. She was supported for this project by U54 CA153460, U54 CA 155496, the Institute for Clinical and Translational Medicine UL1 TR000448 and the Leukemia SPORE P50 CA171963.

\section{Author Contributions}

ECB, LEL, KHM, GC, and SG designed the initial group methodology. ECB, LEL, SC, GF, KF, JH, $\mathrm{KM}$, and $\mathrm{AR}$ all participated in idea generation, discussion, and group observations. ECB and LEL wrote the manuscript. ECB, LEL, SC, GF, KF, JH, KM, AR, and SG outlined and edited manuscript.

\section{References}

[1] Gehlert, S., Murray, A., Sohmer, D., McClintock, M., Conzen, S., \& Olopade, O. (2010). The importance of transdisciplinary collaborations for understand- 
Emily C. Benesh, Laura E. Lamb, Shahnjayla K. Connors, Grant W. Farmer, Katherine C. Fuh, Jean Hunleth, Katherine L. Montgomery, Alex T. Ramsey, Kelle H. Moley, Graham A. Colditz, Sarah J. Gehlert

A Case Study Approach to Train Early-Stage Investigators in Transdisciplinary Research

ing and resolving health disparities. Social Work in Public Health , 25(3-4), 408-422.

[2] Kessel, F., \& Rosenfield, P. L. (2008). Toward transdisciplinary research: historical and contemporary perspectives. American Journal of Preventive Medicine, 35(2), S225-S234.

[3] Hall, K. L., Stokols, D., Moser, R. P., Taylor, B. K., Thornquist, M. D., Nebeling, L. C., ... \& Goran, M. I. (2008). The collaboration readiness of transdisciplinary research teams and centers: findings from the National Cancer Institute's TREC yearone evaluation study. American journal of preventive medicine, 35(2), S161-S172.

[4] Vogel, A. L., Feng, A., Oh, A., Hall, K. L., Stipelman, B. A., Stokols, D., ... \& Nebeling, L. (2012). Influence of a National Cancer Institute transdisciplinary research and training initiative on trainees' transdisciplinary research competencies and scholarly productivity. Translational behavioral medicine,2(4), 459-468.

[5] Patterson, R. E., Colditz, G. A., Hu, F. B., Schmitz, K. H., Ahima, R. S., Brownson, R. C., ... \& Gill, J. (2013). The 20112016 Transdisciplinary Research on Energetics and Cancer (TREC) initiative: rationale and design. Cancer Causes \& Control, 24(4), 695704 .

[6] Gehlert, S., Hall, K., Vogel, A., Hohl, S., Hartman, S., Nebeling, L., ... \& Thompson, B. (2014). Advancing Transdisciplinary Research: The Transdisciplinary Research on Energetics and Cancer Initiative. Journal of translational medicine $\&$ epidemiology, 2(2), 1032.

[7] Cohen, D. S. (2005). The heart of change field guide: Tools and tactics for leading change in your organization. Harvard Business Press. p. 239

[8] Stokols, D., Misra, S., Moser, R. P., Hall, K. L., \& Taylor, B. K. (2008). The ecology of team science: understanding contextual influences on transdisciplinary collaboration. American journal of preventive medicine, 35(2), S96-S115.

[9] Nash, J. M. (2008). Transdisciplinary training: key components and prerequisites for success. American journal of preventive medicine, 35(2), S133-S140.

[10] Canning, C. G., Hird, M., \& Smith, G. (2010). The pitfalls of the "add-and-stir" approach to transdisciplinary public health research. Critical Public Health,20(2), 145-155.

[11] Tools, M. (n.d.). Enhancing Learning through Immediate Application. Case Study Based Learning. Retrieved January 15, 2015, from
http://www.mindtools.com/pages/article/newISS 94.htm.

[12] Hall, K. L., Vogel, A. L., Stipelman, B. A., Stokols, D., Morgan, G., \& Gehlert, S. (2012). A fourphase model of transdisciplinary team-based research: goals, team processes, and strategies. Translational behavioral medicine, 2(4), 415-430.

[13] Hall, K. L., Stokols, D., Stipelman, B. A., Vogel, A. L., Feng, A., Masimore, B., ... \& Berrigan, D. (2012). Assessing the value of team science: a study comparing center-and investigator-initiated grants. American journal of preventive medicine, 42(2), 157163. 
Emily C. Benesh, Laura E. Lamb, Shahnjayla K. Connors, Grant W. Farmer, Katherine C. Fuh, Jean Hunleth, Katherine L. Montgomery, Alex T. Ramsey, Kelle H. Moley, Graham A. Colditz, Sarah J. Gehlert

A Case Study Approach to Train Early-Stage Investigators in Transdisciplinary Research

\section{About the Authors}

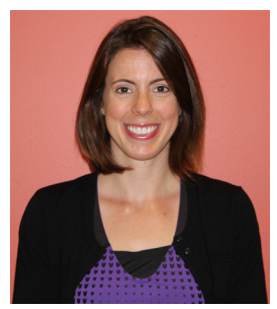

Emily Benesh, PhD (role postdoctoral fellow; developmental biology) is a senior postdoctoral research scholar in the Department of Obstetrics and Gynecology at Washington University School of Medicine in Saint Louis. She is also currently pursuing a certification in Clinical Investigation from the Institute of Clinical and Translational Sciences at Washington University. She did her predoctoral training in the Department of Cell and Developmental Biology at Vanderbilt University. Her research focus is on the role of maternal obesity in generational transmission of cancer and other chronic diseases.

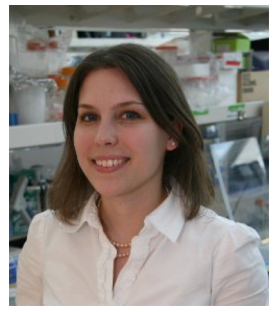

Laura E. Lamb, PhD (role postdoctoral fellow; cell and molecular biology) is an Associate Professor at Oakland University William Beaumont School of Medicine. She did her postdoctoral research on the role of hormone signaling and maternal obesity in cancer at Washington University School of Medicine in St. Louis. Dr. Lamb's current research focuses on determining underlying disease mechanisms and potential biomarkers for interstitial cystitis, radiation cystitis, and underactive bladder.

Shahnjayla K. Connors, PhD, MPH, CPH (role postdoctoral fellow; molecular biology and public health) is an Adjunct Assistant Professor at the University of North Texas Health Science Center. She did her postdoctoral training in the Program for the Elimination of Cancer Disparities (PECaD) at Washington University in St. Louis. Her predoctoral training was in molecular biology from the University of Florida. She obtained an MPH and a Graduate Certificate in Diasporas \& Health Disparities from the University of South Florida. Her research interests include cancer health disparities in minority populations and diversity in the biomedical sciences.

Grant W. Farmer, PhD, MPH (role postdoctoral fellow; epidemiology) was a postdoctoral fellow at Washington University in St. Louis and an Adjunct Professor in the Saint Louis University Center for Outcomes Research. His research interests centered on chronic disease and disease prevention in sexual minority populations, particularly relating to cardiovascular disease and diabetes. He also studied substance use and chronic illness; and social support and disease prevention/management.

Katherine C. Fuh, MD, PhD (role early investigator; medicine and reproductive biology) is an instructor and gynecologic oncologist at Washington University in St. Louis. She received her MD at Georgetown University and $\mathrm{PhD}$ in Cell Biology at Stanford University. Dr. Fuh's research interests include identifying novel targets in ovarian cancer metastasis and developing human and mouse models in ovarian cancer.

Jean Hunleth, PhD, MPH (role postdoctoral fellow; anthropology) is a research scientist in the Division of Public Health Sciences at Washington University School of Medicine in St. Louis. She did her postdoctoral training in community-based cancer disparities research at Washington University and the Siteman Cancer Center. Her research focuses on understanding how people experience and respond to global health inequities in their daily lives, with the goal of improving access to care and informing policy change.

Katherine Montgomery, PhD, MSSW (role postdoctoral fellow, behavior health) is currently the Director at Little Sunshine's Playhouse and Preschool in St. Louis, Missouri. Dr. Montgomery received her MSSW and PhD from the University of Texas at Austin and has authored over 30 publications related to at-risk youth. Dr. Montgomery has worked with children and families for over 15 years, and her work has largely coalesced around the prevention of delinquency through early intervention.

Alex Ramsey, PhD (role postdoctoral fellow; psychology) is a postdoctoral researcher at the George Warren Brown School of Social Work at Washington University in St. Louis. Dr. Ramsey received his doctoral degree in Applied Psychology from Southern Illinois University Carbondale in 2012. Following graduation, he was a Research Associate in the NIDA-funded P30 Center for Technology and Behavioral Health at the Dartmouth Psychiatric Research Center.

Kelle Moley, MD (role mentor; medicine and endocrinology) is the James P. Crane Professor of Obstetrics and Gynecology at the Washington University School of Medicine in St. Louis and Vice Chair for Basic Science Research and Director of the Division of Basic Science Research in the Department of Obstetrics and Gynecology. Dr. Moley is also Co-Director of the Institute of Clinical and Translational Sciences (ICTS), an NIH funded CTSA Institute at Washington University in St. Louis. She is also the PI of the NIH K12 Reproductive Scientist Development Program that now resides at Washington University in St. Louis.

Graham A. Colditz, MD, DrPH (role mentor; epidemiology) is the Niess-Gain professor of Surgery and 
Emily C. Benesh, Laura E. Lamb, Shahnjayla K. Connors, Grant W. Farmer, Katherine C. Fuh, Jean Hunleth, Katherine L. Montgomery, Alex T. Ramsey, Kelle H. Moley, Graham A. Colditz, Sarah J. Gehlert

Associate Director for Prevention and Control at Siteman Cancer Center. He is a cancer epidemiologist and PI on the Transdisciplinary Research on Energetics and Cancer (TREC) at Washington University School of Medicine in St. Louis.

Sarah Gehlert, PhD (role mentor; social science) is the E. Desmond Lee Professor of Racial and Ethnic Diversity at the George Warren Brown School of Social Work and Professor in the Department of Surgery at Washington University in St. Louis. Dr. Gehlert is the Associate Director of the U54 Washington University Transdisciplinary Research in Energetics and Cancer and the Director of its Education, Training, and Outreach Core. From 2003 to 2009, she was Principal Investigator and Director of the P50 Center for Interdisciplinary Health Disparities Research at the University of Chicago.

Copyright (c) 2015 by the authors. This is an open access article distributed under the Creative Commons Attribution License (https://creativecommons.org/licenses/by/4.0/), which permits unrestricted use, distribution, and reproduction in any medium, provided the original work is properly cited. 